\section{Eventos adversos e incidentes sem dano em recém-nascidos notificados no Brasil, nos anos 2007 a 2013}

\author{
Neonatal adverse events and near misses reported \\ in Brazil from 2007 to 2013
}

\author{
Eventos adversos e incidentes sin daño en recién \\ nacidos, notificados en Brasil, durante los años \\ de 2007 a 2013
}

Luciana da Silva Lanzillotti 1

Carla Lourenço Tavares de Andrade 2

Walter Mendes 2

Marismary Horsth De Seta 2

\title{
Resumo
}

O estudo teve o objetivo de analisar os eventos adversos e outros incidentes que não causaram danos em recém-nascidos até 28 dias de vida, notificados no Sistema de Notificações para a Vigilância Sanitária (NOTIVISA) nos anos de 2007 a 2013. Trata-se de estudo quantitativo, descritivo, retrospectivo, com análise de dados secundários. Foram notificados 355 incidentes, desses, 118 (33,3\%) relacionaram-se a artigos médicos, 4 (1,1\%) a equipamentos médicos, e 233 (65,6\%) a medicamentos. No que tange aos medicamentos, o nitrato de prata e os antibióticos foram os mais notificados, e nos artigos e equipamentos médicos, o uso de cateteres venosos como precursores de flebites, o evento adverso mais notificado. O estudo desvela notificações de eventos adversos e de incidentes que não causaram dano, provocando discussões do que efetivamente é considerado dano para aquele que faz a notificação. O desafio para o NOTIVISA é o seu aprimoramento que, como nos demais sistemas de informação, decorre de: uso, crítica e relação com os usuários - notificadores e interessados, tais como as instituições de pesquisa e ensino.

Segurança do Paciente; Recém-Nascido; Vigilância Sanitária;

Efeitos Adversos

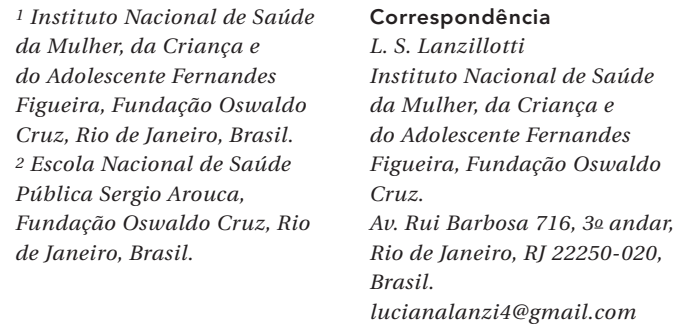

1 Instituto Nacional de Saúde da Mulher, da Criança e do Adolescente Fernandes Figueira, Fundação Oswaldo Cruz, Rio de Janeiro, Brasil. 2 Escola Nacional de Saúde Pública Sergio Arouca, Fundação Oswaldo Cruz, Rio de Janeiro, Brasil.

\author{
Correspondência \\ L. S. Lanzillotti \\ Instituto Nacional de Saúde \\ da Mulher, da Criança e \\ do Adolescente Fernandes \\ Figueira, Fundação Oswaldo \\ Cruz. \\ Av. Rui Barbosa 716, 3o andar, \\ Rio de Janeiro, RJ 22250-020, \\ Brasil. \\ lucianalanzi4@gmail.com
}




\section{Introdução}

Os maiores desafios para a redução da mortalidade infantil no país se concentram no período neonatal (até 28 dias de vida), que responde por $70 \%$ das mortes no primeiro ano de vida, sobretudo no período neonatal precoce (até seis dias de vida), responsável por $52,3 \% 1,2$. O componente neonatal da mortalidade infantil tem sido considerado o de mais difícil redução, especialmente por refletir o cuidado à gestante e ao parto, bem como ao recém-nascido ${ }^{2,3}$. Incidentes podem ocorrer em todos esses processos de atenção, afetando a sua qualidade. Na assistência aos recém-nascidos mais vulneráveis - de mais baixo peso e idade gestacional - as características do próprio cuidado prestado concorrem para a ocorrência de incidentes 4 .

A Classificação Internacional de Segurança do Paciente (International Classification for $\mathrm{Pa}$ tient Safet - ICPS) da Organização Mundial da Saúde (OMS) 5 define um incidente, como um evento ou circunstância que poderia ter resultado ou resultou em dano desnecessário ao paciente, advindos de atos não intencionais ou intencionais.

$\mathrm{O}$ incidente pode atingir ou não o paciente. Caso não atinja, pode ser uma circunstância notificável, ou um near miss. A circunstância notificável está relacionada a um dispositivo médico que poderia provocar um incidente, mas não chegou a ser usado no paciente. O near miss é um incidente que não chegou a atingir o paciente durante o cuidado porque houve alguma intervenção que o evitou. $\mathrm{O}$ incidente, quando atinge o paciente, pode ou não causar dano. Quando não causa dano, é chamado de incidente sem dano e, quando causa, é chamado de evento adverso. Segundo o ICPS, a reação adversa ao medicamento é um dano inesperado que resulta de uma ação ou tratamento medicamentoso justificado e correto para o contexto em que o evento ocorreu.

Diferentes métodos são usados para se detectar e compreender os fatores contribuintes dos incidentes. Em pesquisas, o mais utilizado tem sido a revisão retrospectiva de prontuários. Entretanto, nos serviços de saúde, informações obtidas em sistemas de notificação são muito utilizadas, embora existam limitações das informações provenientes de sistemas de notificação voluntária 6 , que decorrem do viés retrospectivo da análise do incidente, da incompletude dos dados e da subnotificação. A cultura de culpabilizar os profissionais envolvidos em eventuais erros pode explicar, em parte, a subnotificação.

A despeito do reconhecimento dessas limitações 7, esforços têm sido despendidos para a construção de sistemas de notificação voluntária de eventos adversos em vários países 8 . As informações procedentes dos sistemas de notificação permitem a identificação de condições ativas e latentes que contribuem para o surgimento dos incidentes. As falhas latentes, detectáveis particularmente mediante os sistemas de notificação, expressam características da estrutura e do processo de cuidado e podem se relacionar ao ambiente e às condições em que é prestado o cuidado ${ }^{9}$, incluindo a segurança dos insumos utilizados. E a disponibilidade dos seus relatórios acarreta que sejam fonte atraente de pesquisa de incidentes 7, que a maioria das instituições de saúde utiliza 8 .

No Sistema Único de Saúde (SUS) do Brasil, o Sistema de Notificações para a Vigilância Sanitária (NOTIVISA) é um sistema informatizado da Agência Nacional de Vigilância Sanitária (Anvisa) que registra incidentes, inclusive eventos adversos, e queixas técnicas relativas a tecnologias, produtos e processos relacionados à assistência 10 , de forma voluntária e anônima. As notificações no NOTIVISA podem ser feitas pelos profissionais de saúde, cidadãos, organizações e serviços de saúde, ou ainda pelas empresas detentoras de registro de produtos sob vigilância sanitária, fabricantes, importadores e distribuidores ${ }^{11}$. As informações contidas no NOTIVISA subsidiam o Sistema Nacional de Vigilância Sanitária (SNVS), que promove ações de proteção à saúde dos cidadãos; regula a produção, registro e comercialização de insumos utilizados no processo de atenção e outros de interesse sanitário; e contribui para a prestação de serviços de saúde mais seguros.

O NOTIVISA é um sistema de implantação relativamente recente e alguns estudos foram realizados sobre ele ou com base nas suas informações 12,13,14, contudo, sem que se destinassem à análise dos registros referentes aos incidentes em pacientes no período neonatal. Ao mesmo tempo, são relativamente escassos os estudos nacionais sobre incidentes e eventos adversos em recém-nascidos 4 .

Este estudo tem como objetivo analisar os eventos adversos e outros incidentes que não causaram danos em recém-nascidos até 28 dias de vida, notificados no NOTIVISA nos anos de 2007 a 2013, no Brasil. Espera-se que ele possa contribuir para a prevenção da ocorrência dos incidentes no cuidado aos recém-nascidos e, consequentemente, para a melhoria da qualidade da assistência que lhes é prestada. 


\section{Método}

Trata-se de um estudo quantitativo, descritivo, retrospectivo, com análise de dados secundários registrados no NOTIVISA, desde a sua implantação, em 2007, até setembro de 2013. Solicitaram-se as informações sobre os eventos adversos notificados envolvendo recém-nascidos nos primeiros 28 dias de vida diretamente ao responsável pelo NOTIVISA. Três bases de dados foram disponibilizadas para a pesquisa: de artigos médicos, de equipamentos médicos e a relacionada a medicamentos.

A qualidade dos dados recebidos foi avaliada mediante o critério de "incompletude", aplicado em outros estudos 12,15, levando em conta a proporção de informação ignorada, nos campos em branco. Os escores utilizados para avaliação da qualidade da informação foram os seguintes: excelente (menos de $5 \%$ de campos não preenchidos), bom (entre $5 \%$ e $10 \%$ ), regular (entre $10 \%$ e $20 \%$ ), ruim (entre $20 \%$ e $50 \%$ ) e muito ruim $(50 \%$ ou mais) 12,15 . Outros critérios de avaliação de qualidade dos dados também foram observados, como: a acessibilidade (disponibilidade dos dados), oportunidade (tempo de entrega), clareza metodológica (manuais, conceitos e definições) e a consistência (análise da plausibilidade dos resultados) 15, e serviram também para a definição do objeto em estudo.

As variáveis avaliadas foram as seguintes: número total de notificações, produto notificado, descrição do incidente/motivo da notificação, idade, sexo, Unidade da Federação e dano causado.

Na definição encontrada no NOTIVISA, um evento adverso relacionado a medicamento refere-se ao caso em que existe uma suspeita de que o dano sofrido pelo paciente ou usuário tenha ocorrido após a utilização de um medicamento. Evento adverso relacionado a medicamento é, portanto, uma definição mais abrangente do que a estabelecida internacionalmente para reação adversa ao medicamento, no contexto da farmacovigilância. Essa representa toda resposta nociva e não intencional a um medicamento relacionada a qualquer dose, em que há uma possível ou provável relação causal entre o uso do medicamento e a resposta apresentada.

Em relação aos eventos relacionados a medicamentos, dois farmacêuticos especialistas reclassificaram os incidentes classificados como reação adversa ao medicamento no NOTIVISA, utilizando o conceito da OMS. Os farmacêuticos avaliaram cada caso de reação adversa ao medicamento separadamente com consulta bibliográfica da área e exploraram os seguintes itens: princípio ativo; descrição do incidente; presença da descrição do incidente na bula e/ou na literatura nacional e internacional; relação causal entre o medicamento e o incidente. Após essa primeira análise feita individualmente, os dois farmacêuticos se reuniram para discutir as divergências e definir de modo consensual cada caso avaliado. Os incidentes reclassificados como "não reação adversa ao medicamento" pelos especialistas foram considerados neste estudo como um evento adverso.

Procedimento análogo foi adotado para os eventos relacionados a artigos e equipamentos médicos. Dois profissionais da área de segurança do paciente (um médico e uma enfermeira) reclassificaram os incidentes do NOTIVISA como: sem dano ou evento adverso, e avaliaram a descrição do incidente, a causa e a consequência. Dessa forma, se o incidente não ocasionou danos ao recém-nascido foi classificado como incidente sem dano, caso contrário, como evento adverso. Optou-se por classificar os fatores contribuintes desses incidentes em três categorias: problemas no manejo; problemas na qualidade; problemas no manejo e/ou na qualidade do artigo ou do equipamento médico.

Realizou-se análise descritiva com distribuição de frequências apresentadas em tabelas e teste qui-quadrado dos incidentes relacionados à idade do recém-nascido. A significância estatística de tal associação foi verificada pelo valor de $\mathrm{p}<0,05$.

Aspectos éticos foram verificados segundo a Resolução no 466/2012, do Conselho Nacional de Saúde, pois, apesar de se tratar de uma investigação com dados secundários, envolve informações de seres humanos, tanto os que sofreram os eventos adversos notificados, quanto os profissionais que os notificaram. Nas bases de dados recebidas, não constava a identificação das instituições notificadoras. De todo modo, para impossibilitar a identificação dos fabricantes, marcas registradas e pacientes, houve o compromisso de confidencialidade e codificaram-se os dados das pessoas e dos produtos e serviços envolvidos. A pesquisa obteve aprovação do Comitê de Ética em Pesquisa da Escola Nacional de Saúde Pública Sergio Arouca, Fundação Oswaldo Cruz (ENSP/ Fiocruz) sob número de parecer no 486.254.

\section{Resultados}

Foram notificados 355 incidentes ocorridos em recém-nascidos até 28 dias de vida, no NOTIVISA, entre janeiro de 2007 e setembro de 2013. Desses, 118 (33,3\%) relacionaram-se a artigos médicos, 4 $(1,1 \%)$ a equipamentos médicos, e $233(65,6 \%)$ a medicamentos. A base de equipamentos e a de 
artigos médicos apresentaram motivos de notificação similares, por isso, foram unificados em um mesmo banco para análise.

O NOTIVISA é acessível, a base de dados foi obtida após solicitação por meio eletrônico, apesar de nela não constarem os estabelecimentos em que ocorreram os incidentes. Pode ser considerado oportuno, devido à entrega em dois meses após a solicitação, e com uma defasagem de apenas seis meses entre a visualização da notificação e a disponibilização do dado. Existe relativa clareza metodológica quando se analisam os manuais disponíveis visto que constam definições das variáveis, porém não se encontraram comentários sobre os campos que não são preenchidos. Quanto à consistência, que reflete a qualidade das variáveis, recomenda-se ser revista, sobretudo na notificação de incidentes sem dano como eventos adversos e no banco de medicamentos, o que tornou necessário sua reclassificação por especialistas.

No que tange à completude dos dados, na análise quantitativa, a qualidade dos bancos mostrou-se deficiente. Na base de dados relacionada a incidentes com artigos e equipamentos médicos, o grau de incompletude alcançou $44,2 \%$, considerando a soma dos critérios "muito ruim" (33,8\%) e "ruim" (10,4\%). Quanto à base referente a medicamentos, a qualidade foi pior, perfazendo $68,2 \%$ a soma dos critérios "muito ruim" (37,9\%) e "ruim" (30,3\%). Contudo, essa deficiência quantitativa na completude dos bancos, não inviabilizou a utilização dos dados, pois a descrição dos incidentes era satisfatória.

Aconteceu um crescimento gradual do número de notificações referentes ao período neonatal, tendo em vista os anos pesquisados. Em 2007, não havia notificações sobre incidentes relacionados a medicamentos, somente 8 relacionados a artigos e equipamentos médicos. Em 2008, foram notificados 57 incidentes de medicamentos e 8 de artigos e equipamentos médicos; em 2009, foram 24 incidentes de medicamentos e 4 de artigos e equipamentos médicos; em 2010, foram 54 incidentes de medicamentos e 4 de artigos e equipamentos médicos; em 2011, foram 17 incidentes de medicamentos e 13 de artigos e equipamentos médicos; em 2012, 54 incidentes de medicamentos e 54 de artigos e equipamentos médicos; e até setembro de 2013, 57 incidentes de medicamentos e 31 de artigos e equipamentos médicos.

\section{Incidentes relacionados com artigos e equipamentos médicos}

Vinte e três dias foi o tempo médio decorrido entre o incidente e as notificações envolvendo artigos e equipamentos médicos. O Estado de São Paulo (n = 70; 19,2\%) e o Estado da Paraíba ( $n=52,14,8 \%$ ) foram os que mais notificaram.

Os principais fatores contribuintes para os 122 incidentes relacionados aos artigos e equipamentos médicos foram: 55 (45,1\%) referentes a problemas com cateteres centrais de inserção periférica (PICC, peripherally inserted central venous catheter), 22 (18\%) relacionados ao cateter venoso periférico e $16(13,1 \%)$ relacionados ao vazamento do equipo de soro.

Constatou-se que em 77 (63,1\%) dos incidentes ocasionou um dano (eventos adversos) e em $45(36,9 \%)$ não se encontraram danos. Não foram detectadas circunstâncias notificáveis nem near misses.

Com base no registro dos incidentes, detalharam-se a frequência e os fatores contribuintes dos eventos adversos. Quando havia informação disponível, foi descrita a consequência dos danos nos recém-nascidos ou das ações realizadas após detecção do problema (Tabela 1). Destacaram-se como os eventos adversos de maior frequência: a flebite, o hematoma, o edema e a lesão de pele.

Na Tabela 2, estão descritos os incidentes sem dano, sua frequência, os fatores contribuintes e, quando disponíveis, a consequência nos recémnascidos ou das ações realizadas após detecção do problema. O incidente sem dano mais frequente foi a "perda de acesso venoso profundo" ( $\mathrm{n}=28 ; 62,2 \%$ ).

Na Tabela 3, são apresentadas a descrição da classificação e a frequência dos fatores contribuintes dos incidentes sem dano e dos eventos adversos relacionados a artigos e equipamentos médicos.

Dos artigos ou equipamentos informados, 61 (50\%) eram de fabricação nacional proveniente especialmente do Estado de São Paulo. Um dado chama a atenção: 114 (93,4\%) usuários ou profissionais de saúde relataram terem sido seguidas as instruções do fabricante do produto.

Na Tabela 4, encontra-se descrita a frequência dos incidentes sem dano e dos eventos adversos, segundo a idade dos recém-nascidos. Evidencia-se que 45 (36,9\%) incidentes ocorreram no período neonatal precoce, com significância estatística. Os recém-nascidos no período neonatal precoce que sofreram eventos adversos perfazem $35(44,2 \%)$, sendo relevante destacar casos como: extravasamento de soluções do cateter umbilical no peritônio, causando abdome agudo, asfixia por causa da "dobra" de tubo orotraqueal, queimadura gerada por solução alcoólica aplicada em recém-nascidos prematuro extremo, conduzindo a antibioticoterapia, flebites no uso PICC, entre outros. 
Descrição e frequência dos eventos adversos relacionados a artigos e equipamentos médicos e apresentação dos fatores contribuintes e consequências nos recém-nascidos registrados no NOTIVISA de 2007 até setembro de 2013.

\begin{tabular}{|c|c|c|c|c|}
\hline \multirow[t]{3}{*}{ Eventos adversos/Fatores contribuintes } & \multirow[t]{3}{*}{ Consequências } & \multicolumn{3}{|c|}{ Total } \\
\hline & & \multicolumn{2}{|c|}{$\mathrm{n}$} & \multirow[t]{2}{*}{$\%$} \\
\hline & & Parcial & Total & \\
\hline \multicolumn{5}{|l|}{ Flebite } \\
\hline Problemas no uso do PICC & Retirado PICC & 16 & 20 & 26,0 \\
\hline Problemas com o cateter venoso periférico & & 3 & & \\
\hline Múltiplas punções venosas & & 1 & & \\
\hline \multicolumn{5}{|l|}{ Hematoma } \\
\hline Múltiplas punções venosas & - & 12 & 12 & 15,6 \\
\hline \multicolumn{5}{|l|}{ Edema } \\
\hline \multirow[t]{2}{*}{ Dificuldade na progressão de PICC } & Retirado PICC e indicada dissecção & 7 & 11 & 14,3 \\
\hline & venosa & & & \\
\hline PICC perfurado & - & 4 & & \\
\hline \multicolumn{5}{|l|}{ Lesão de pele } \\
\hline Remoção de eletrodo & - & 3 & 9 & 11,6 \\
\hline Múltiplas punções venosas & - & 6 & & \\
\hline \multicolumn{5}{|l|}{ Lesão de septo nasal } \\
\hline \multirow[t]{2}{*}{ Problemas no uso do CPAP nasal } & Intubação orotraqueal & 1 & 4 & 5,2 \\
\hline & - & 3 & & \\
\hline \multicolumn{5}{|l|}{ Sangramento } \\
\hline Rompimento de balão de sonda uretral & Lesão uretral & 1 & 4 & 5,2 \\
\hline Problemas com o clamp umbilical & Queda de parâmetros clínicos & 1 & & \\
\hline Problemas em conectores de acesso venoso & Transfusão sanguínea & 2 & & \\
\hline \multicolumn{5}{|l|}{ Queimadura } \\
\hline Uso de clorexidina alcoólica na pele de prematuro extremo & $\begin{array}{c}\text { Alteração de hemograma e início de } \\
\text { antibiótico }\end{array}$ & 1 & 4 & 5,2 \\
\hline Problemas com bisturi elétrico & Amputação & 1 & & \\
\hline Problemas no uso do sensor de monitorização & - & 1 & & \\
\hline Problemas no uso da fototerapia & - & 1 & & \\
\hline \multicolumn{5}{|l|}{ Asfixia } \\
\hline Dobradura do TOT & Hipertensão pulmonar & 1 & 3 & 3,9 \\
\hline Problemas na desconexão do mandril no TOT & Hipoxia e cianose & 1 & & \\
\hline Aderência da sonda em tubo orotraqueal & - & 1 & & \\
\hline \multicolumn{5}{|l|}{ Presença de fragmento de cateter periférico partido dentro da veia } \\
\hline Problemas com o cateter venoso periférico & & 3 & 3 & 3,9 \\
\hline \multicolumn{5}{|l|}{ Hipotensão } \\
\hline Problemas em conectores de acesso venoso & - & 2 & 2 & 2,6 \\
\hline \multicolumn{5}{|l|}{ Óbito } \\
\hline Problemas com cateter venoso profundo & - & 1 & 2 & 2,6 \\
\hline Problemas na bomba infusora & - & 1 & & \\
\hline \multicolumn{5}{|l|}{ Parada cardiorrespiratória } \\
\hline Extubação pela não aderência do esparadrapo entre o TOT e a pele & - & 1 & 1 & 1,3 \\
\hline \multicolumn{5}{|l|}{ Abdome agudo } \\
\hline Cateter umbilical extravasou as infusões para o peritônio & - & 1 & 1 & 1,3 \\
\hline \multicolumn{5}{|l|}{ Hiperglicemia } \\
\hline Problemas na bomba infusora & Piora do quadro clínico & 1 & 1 & 1,3 \\
\hline Total & & 77 & & 100,0 \\
\hline
\end{tabular}

CPAP: continuous positive airway pressure (pressão positiva nas vias aéreas); PICC: peripherally inserted central catheter (cateter central de inserção periférica); TOT: tubo orotraqueal.

Fonte: Agência Nacional de Vigilância Sanitária. Sistema de Notificação em Vigilância Sanitária - NOTIVISA 2007 a 2013. 
Tabela 2

Descrição e frequência dos Incidentes sem dano relacionados a artigos e equipamentos médicos, fatores contribuintes e consequências nos recém-nascidos registrados no NOTIVISA de 2007 até setembro de 2013.

\begin{tabular}{|c|c|c|c|c|}
\hline \multirow[t]{3}{*}{ Incidente sem dano/Fatores contribuintes } & \multirow[t]{3}{*}{ Consequências } & \multicolumn{3}{|c|}{ Total } \\
\hline & & \multicolumn{2}{|c|}{$\mathbf{n}$} & \multirow[t]{2}{*}{$\%$} \\
\hline & & Parcial & Total & \\
\hline \multicolumn{5}{|l|}{ Perda de acesso venoso profundo } \\
\hline Rompimento de cateter & Submissão a um novo procedimento invasivo para & 17 & 28 & 62,2 \\
\hline Problemas em conectores & acesso venoso central & 4 & & \\
\hline Perfurações de cateter & & 3 & & \\
\hline Não progressão & & 2 & & \\
\hline Dobra de cateter & & 1 & & \\
\hline Dificuldade de tração do cateter no vaso & & 1 & & \\
\hline \multicolumn{5}{|l|}{ Vazamento de equipo de soro } \\
\hline Rachadura ou rompimento do equipo & Suspensão do uso de NPT & 15 & 15 & 33,3 \\
\hline \multicolumn{5}{|l|}{ Clamp umbilical quase solto } \\
\hline Facilidade de abertura de clamp umbilical & - & 1 & 1 & 2,2 \\
\hline \multicolumn{5}{|l|}{ Desconexão de tubos e sondas } \\
\hline Esparadrapo com baixa adesividade & Repetição de procedimentos invasivos & 1 & 1 & 2,2 \\
\hline Total & & \multicolumn{2}{|c|}{45} & 100,0 \\
\hline
\end{tabular}

NPT: nutrição parenteral total

Fonte: Agência Nacional de Vigilância Sanitária. Sistema de Notificação em Vigilância Sanitária - NOTIVISA 2007 a 2013.

Tabela 3

Classificação dos fatores contribuintes do incidente e sua frequência referente aos incidentes sem dano e a eventos adversos relacionados a artigos e equipamentos médicos registrados no NOTIVISA de 2007 até setembro de 2013.

\begin{tabular}{|c|c|c|c|c|c|c|}
\hline \multirow[t]{3}{*}{ Fatores contribuintes } & \multicolumn{4}{|c|}{ Parcial } & \multirow{2}{*}{\multicolumn{2}{|c|}{$\begin{array}{c}\text { Total } \\
\text { Incidente sem dano e eventos } \\
\text { adversos }\end{array}$}} \\
\hline & \multicolumn{2}{|c|}{ Incidente sem dano } & \multicolumn{2}{|c|}{ Eventos adversos } & & \\
\hline & $\mathbf{n}$ & $\%$ & $\mathbf{n}$ & $\%$ & $\mathrm{n}$ & $\%$ \\
\hline Problemas no manejo e/ou qualidade & 24 & 53,3 & 51 & 66,2 & 75 & 61,5 \\
\hline Problemas no manejo & 2 & 4,5 & 2 & 2,6 & 4 & 3,3 \\
\hline Problemas na qualidade & 19 & 42,2 & 24 & 31,2 & 43 & 35,2 \\
\hline Total & 45 & 100,0 & 77 & 100,0 & 122 & 100,0 \\
\hline
\end{tabular}

Fonte: Agência Nacional de Vigilância Sanitária. Sistema de Notificação em Vigilância Sanitária - NOTIVISA 2007 a 2013.

Em 69 (56,4\%) incidentes, foram tomadas providências após a identificação da ocorrência, sendo as mais frequentes: suspensão do uso do produto, notificação e busca do produto nos demais setores do hospital. A comunicação à indústria fabricante ocorreu em 58 (47,5\%) casos, principalmente por e-mail ou por comunicação ao representante da fábrica.

\section{Incidentes relativos a medicamentos}

As notificações relativas a medicamentos foram avaliadas em média em 74 dias após a notificação. Os princípios ativos mais notificados foram o nitrato de prata em 66 (28,3\%) casos e os antibióticos, especialmente benzilpenicilina e vancomicina, em 64 (27,3\%) casos.

Dos 233 incidentes analisados, 54\% $(n=127)$ foram classificados como reação adversa ao 
Frequência de incidentes sem dano e eventos adversos associados à idade do recém-nascido referente a artigos e equipamentos médicos registrados no NOTIVISA de 2007 até setembro de 2013

\begin{tabular}{|c|c|c|c|c|c|c|c|}
\hline \multirow[t]{3}{*}{ Idade do recém-nascido } & \multicolumn{4}{|c|}{ Tipo de incidente } & \multicolumn{2}{|c|}{ Total } & \multirow{3}{*}{$\begin{array}{c}\text { Valor de } \\
\qquad p^{\star}\end{array}$} \\
\hline & \multicolumn{2}{|c|}{ Sem dano } & \multicolumn{2}{|c|}{ Evento adverso } & \multirow[b]{2}{*}{$\mathbf{n}$} & \multirow[b]{2}{*}{$\%$} & \\
\hline & n & $\%$ & $\mathrm{n}$ & $\%$ & & & \\
\hline Recém-nascido no período neonatal precoce (até o sexto dia de vida) & 11 & 24,4 & 34 & 44,2 & 45 & 36,9 & 0,034 \\
\hline Recém-nascido com mais de uma semana de vida & 34 & 75,6 & 43 & 55,8 & 77 & 63,1 & \\
\hline Total & 45 & 100,0 & 77 & 100,0 & 122 & 100,0 & \\
\hline
\end{tabular}

* Teste qui-quadrado

Fonte: Agência Nacional de Vigilância Sanitária. Sistema de Notificação em Vigilância Sanitária - NOTIVISA 2007 a 2013.

medicamento, 32\% ( $\mathrm{n}=74)$ como indefinidos e $14 \%$ ( $\mathrm{n}=32$ ) como eventos adversos (não reação adversa ao medicamento).

A Tabela 5 apresenta a descrição de suas frequências de acordo com o registro no NOTIVISA da gravidade do dano causado no recém-nascido. Nota-se um maior percentual associado a consequências não graves, como o uso do nitrato de prata que conduziu a uma secreção ocular anormal $(41,2 \% ; n=96)$ e efeitos clinicamente importantes, como o uso de antibiótico associado à inefetividade do medicamento $(37,3 \%$; $\mathrm{n}=87$ ). Ressaltam-se dois casos de óbitos e sete casos de ameaças à vida relacionados à eventos adversos. Os eventos adversos relacionados ao uso de medicamentos foram, sobretudo, erros de dosagem.

O Estado de São Paulo e da Paraíba, com $46,8 \%$ e $11,2 \%$ foram, respectivamente, os que mais notificaram.

Os recém-nascidos que tiveram incidentes com medicamentos têm o seguinte perfil: $31,6 \%$ ( $\mathrm{n}=99)$ do sexo feminino e $25,2 \%(\mathrm{n}=79)$ do sexo masculino. Desses, 118 (50,6\% dos casos) encontravam-se com peso entre $1.000 \mathrm{~g}$ e $2.500 \mathrm{~g}$, e recém-nascido até o sexto dia de vida somam $14,2 \%(n=33)$. Em 21,5\% ( $=50)$ dos casos, foram adotadas providências após a identificação do problema, como, por exemplo, a suspensão do uso dos medicamentos suspeitos.

\section{Discussão}

A tendência ao aumento das notificações no NOTIVISA a cada ano é também assinalada em outros estudos 13,14. Uma possível causa para essa tendência pode ser a atuação da Rede Brasileira de Hospitais Sentinela, construída pari passu com esse sistema pela Anvisa. Essa rede conta com 193 hospitais que atuam como observatórios do desempenho e segurança de produtos de saúde e são incentivados a notificar eventos adversos no NOTIVISA 16.

São Paulo foi o estado que realizou mais notificações tanto de artigos e equipamentos médi$\cos (19,7 \%)$, quanto de medicamentos $(46,8 \%)$, e é o estado com o maior número de hospitaissentinela - representa $28 \%$ da rede. A Região Sudeste representa $48,7 \%$ de todos os hospitaissentinela 16. Reitera-se a situação da Paraíba que com apenas seis hospitais-sentinela, ocupa o segundo lugar nas notificações, o que pode ser também decorrente de ser esse o estado que primeiro montou uma agência estadual, com uma atuação relativamente estruturada na vigilância sanitária de serviços de saúde.

A partir da implantação do Programa Nacional de Segurança do Paciente 17, tornou-se obrigatória a criação de um núcleo de segurança do paciente em hospitais e serviços de saúde, reforçando a notificação mensal de eventos adversos associados à saúde 16. Essa norma tende também a favorecer o incremento do número de notificações, fato esse visto de modo positivo, pois pode contribuir para estratégias de prevenção dos incidentes, sobretudo dos eventos adversos. Entretanto, a qualidade dos dados no que concerne à incompletude precisa melhorar. Cerca de $45 \%$ dos dados no banco de artigos e equipamentos médicos não foram considerados bons, e no banco de medicamentos esse número chegou a $68,2 \%$. É importante que, ao mesmo tempo em que se estimulam os serviços de saúde a notificar os incidentes, haja também preenchimento dos campos de informações. Deve-se evitar a incompletude dos dados, para adquirir o detalhamento das informações dos incidentes e, assim, favorecer a prevenção dos mesmos. Nos incidentes relacionados a medicamentos, a inclusão da 
Tabela 5

Frequência da gravidade do dano causado nos recém-nascidos de acordo com o tipo de incidente identificado no banco de medicamentos registrados no NOTIVISA de 2007 até setembro de 2013.

\begin{tabular}{|c|c|c|c|c|c|c|c|c|}
\hline \multirow[t]{2}{*}{ Gravidade } & \multicolumn{2}{|c|}{ Reação adversa ao medicamento } & \multicolumn{2}{|c|}{ Indefinido } & \multicolumn{2}{|c|}{ Evento adverso } & \multicolumn{2}{|c|}{ Total } \\
\hline & $\mathbf{n}$ & $\%$ & $\mathrm{n}$ & $\%$ & $\mathbf{n}$ & $\%$ & $\mathbf{n}$ & $\%$ \\
\hline Não grave & 73 & 57,5 & 20 & 27,0 & 3 & 9,4 & 96 & 41,2 \\
\hline Efeito clinicamente importante & 44 & 34,6 & 30 & 40,5 & 13 & 40,6 & 87 & 37,3 \\
\hline Motivou ou prolongou a hospitalização & 3 & 2,4 & 5 & 6,8 & 7 & 21,9 & 15 & 6,4 \\
\hline Incapacidade persistente ou significante & - & - & 1 & 1,4 & - & - & 1 & 0,4 \\
\hline Ameaça a vida & 6 & 4,7 & 16 & 21,6 & 7 & 21,9 & 29 & 12,5 \\
\hline Óbito & 1 & 0,8 & 2 & 2,7 & 2 & 6,2 & 5 & 2,2 \\
\hline Total & 127 & 100,0 & 74 & 100,0 & 32 & 100,0 & 233 & 100,0 \\
\hline
\end{tabular}

Fonte: Agência Nacional de Vigilância Sanitária. Sistema de Notificação em Vigilância Sanitária - NOTIVISA 2007 a 2013.

história clínica e exames complementares, sobretudo a apresentação do medicamento, forma farmacêutica, via de administração, diluição e velocidade de infusão, são informações úteis para o entendimento do incidente.

A classificação dos incidentes - sem dano ou eventos adversos - poderia ser mais explorada no sistema de notificação com o intuito de se aproximar mais da realidade vivenciada nos serviços de saúde. Nem todos os incidentes notificados como eventos adversos causaram danos nos recém-nascidos, que nesta pesquisa foram classificados como incidentes sem dano. $\mathrm{O}$ fato de não ter causado dano não diminui a importância de sua investigação para evitar novas ocorrências. No banco de artigos e equipamentos médicos, $36,9 \%$ dos casos foram classificados como incidentes sem danos. As informações contidas nos bancos, mesmo com os problemas de qualidade dos dados, fornecem elementos essenciais à elaboração de estratégias nacionais e/ou regionais para melhoria da qualidade nos serviços de saúde.

No banco de artigos e equipamentos, a flebite foi o eventos adversos mais notificado causado pelo manuseio de cateteres venosos periféricos e sobremaneira os profundos. A flebite é uma inflamação das células endoteliais da parede venosa que podem provocar edema, hiperemia local, aumento da temperatura basal, cordão fibroso palpável no trajeto da veia e lentidão no fluxo de infusão. A flebite pode ter origens diversas: (i) mecânica quando provocada pela movimentação do cateter na parede íntima da veia, como nos casos de manipulação errônea e má fixação; (ii) química causada pela administração de soluções irritantes e de diluições inapropriadas nesse caso mais encontrado no uso de cateteres periféricos; (iii) origem infecciosa causada pela falha na aplicação da técnica asséptica dos procedimentos ou na manipulação errônea 18.

A ocorrência da flebite pode ser minimizada por intermédio da indicação de acessos venosos centrais para a infusão de fármacos com características físico-químicas inadequadas para infusão nas veias periféricas. Quando o acesso central não for possível, o uso das veias periféricas exige vigilância contínua pela equipe de enfermagem 19.

Outros eventos adversos frequentes foram as lesões de pele e hematomas causados pelas múltiplas punções venosas decorrentes de problemas de qualidade com cateteres venosos periféricos. Estudos 20,21 afirmam que tais eventos adversos estão relacionados à deficiência na assistência de enfermagem, e pouco se discute sobre a qualidade do material, verificado no registro das notificações como fator contribuinte dessas ocorrências - um exemplo virtuoso de detecção de condição latente, que demonstra a validade do trabalho com notificações voluntárias para a prevenção de eventos adversos.

Os incidentes com e sem dano estiveram relacionados majoritariamente a problemas no manejo e na qualidade do PICC. O uso de PICC na neonatologia é cada vez mais frequente e indispensável, sobretudo naqueles neonatos de muito baixo peso. O PICC é um acesso venoso estável e eficaz, indicado quando se necessita de uma veia pérvia num período superior a uma semana, pois evita múltiplas punções e garante a infusão de uma variedade de terapias, como: antibióticos, nutrição parenteral total (NPT) e drogas vasoativas 22,23. A pesquisa revelou que $o$ seu uso resultou em lesões como: flebites (26\%) e edema $(14,3 \%)$. Entre os incidentes sem danos, 
a ocorrência do rompimento do cateter se destacou. As consequências no recém-nascido mais frequentes foram a retirada do PICC, a necessidade de um novo procedimento invasivo e em alguns casos, a dissecção venosa.

Os incidentes relacionados ao PICC também são atribuídos a uma inadequação na técnica asséptica e na manipulação do dispositivo. Desse modo, indica-se treinamento dos profissionais que manipulam o cateter não somente para evitar a ocorrência desses incidentes, mas também para saber como intervir diante dos problemas instalados 24 .

As lesões de pele e a submissão a um novo procedimento invasivo, sobretudo as dissecções venosas, podem levar à infecção hospitalar, considerada uma das causas mais comuns de mortalidade neonatal 25. A infecção é o tipo de eventos adversos que causa maiores danos ao recém-nascido 4 .

O período neonatal precoce é um momento crítico, 44,2\% dos eventos adversos aconteceram nesse período. O recém-nascido, nesse período, sofre a influência das condições da gestação, do parto, e do próprio cuidado neonatal, quando ocorrem $52,3 \%$ dos óbitos no primeiro ano de vida, e se pode chegar a $64,9 \%$, se forem considerados apenas aqueles recém-nascido com muito baixo peso (inferior a $1.500 \mathrm{~g}$ ) 2,26. Trata-se do período mais crítico para a sobrevida do recém-nascido e quando se encontram registros de eventos adversos que causam danos mais graves como: sangramento, queimadura extensa, flebites e asfixia.

As notificações sobre artigos e equipamentos médicos, no período neonatal precoce, somaram $36,9 \%$, e as relativas a medicamentos atingiram 14,9\% dos casos, com tendência a aumento gradual a cada ano. Na notificação de medicamentos, metade do número de casos está relacionada a recém-nascido de baixo peso e de muito baixo peso (entre $1.000 \mathrm{~g}$ e $2.500 \mathrm{~g}$ ). Apesar das diferenças, todas as categorias de baixo peso ao nascer são consideradas de maior risco de morte neonatal 27.

Os incidentes descritos no banco de artigos e equipamentos poderiam ser evitados com a adoção de estratégias de criação e implementação de protocolos; programas de capacitação em serviço, entre outras ações. A flebite, por exemplo, que na maioria das vezes foi oriunda de falha no manejo é um eventos adversos que pode ser prevenido por programas permanentes de capacitação em serviço ${ }^{14}$.

Quando se optou por avaliar os dados do banco de medicamentos utilizando os conceitos oriundos da área da segurança do paciente da OMS foi possível ratificar casos nomeados como reação adversa ao medicamento e considerar outros como evento adverso. Assim, o número de eventos adversos ocorridos nas UTI neonatais aumentou. É válido ressaltar que os incidentes com medicamentos são os mais notificados, dentre todos os produtos sob controle e competência da vigilância sanitária brasileira 14. Também são os mais frequentes entre os recém-nascidos internados em unidades de terapia intensiva neonatal descritos na literatura internacional 4 .

Oserrosdedosagemforamosincidentesmedicamentosos mais frequentes. Alguns estudos 28,29 relacionam os erros de dosagem à falta de medicamentos adequados para a faixa etária 28 . A falta de medicamentos intravenosos disponíveis para o uso pediátrico obriga os serviços de saúde a diluírem os medicamentos 30 , o que favorece o acontecimento dos erros. Vários produtos específicos e adequados para o uso em pediatria já são comercializados em outros países, o que aumenta a urgência da obrigatoriedade desse tipo de produtos prontos no mercado 28 .

As causas de incidentes relacionados aos medicamentos não se restringem à qualidade do produto. Abrangendo também o processo do cuidado, essas causas são comuns a outros países: deficiências na formação dos pediatras para o uso seguro dos medicamentos, problemas estruturais nas farmácias hospitalares e atuação insatisfatória dos farmacêuticos 28 . A maioria dos erros de medicamentos poderia ser evitada com um trabalho multiprofissional, envolvendo o farmacêutico, o médico e o enfermeiro ${ }^{30}$, assim como a mudança da cultura organizacional, com ênfase no cuidado seguro 31 .

O uso do nitrato de prata e de antibióticos, particularmente a benzilpenicilina e a vancomicina, foram os princípios ativos mais relacionados com os incidentes medicamentosos notificados. O Ministério da Saúde recomenda o uso do nitrato de prata a $1 \%$ na prevenção da conjuntivite neonatal, que deve ser administrado no momento do parto, quando existe contato com secreções contaminadas da genital materna 32 . $\mathrm{O}$ nitrato de prata pode causar uma conjuntivite química que, em geral, desaparece em 48 horas 33 . Esse fato pode ser o motivo das notificações mais frequentes, como a secreção ocular anormal e o edema, considerados incidentes não graves. A solução de nitrato de prata é instável, pois sua concentração aumenta em função da evaporação e se modifica pela ação do calor e da luz resultando em uma solução fortemente cáustica ${ }^{34}$. As condições de armazenamento desse fármaco são fundamentais para a prevenção de eventos adversos relacionados ao seu uso.

Os antibióticos, usados rotineiramente nas UTI, são os medicamentos mais relacionados 
com os incidentes. Os pacientes geralmente usam esquemas terapêuticos com dois ou mais antibióticos 35. A imaturidade dos sistemas fisiológicos dos recém-nascidos altera a farmacocinética e a farmacodinâmica de diversos fármacos e contribui com as complicações. Neste estudo, os antibióticos estiveram em grande parte associados à pouca efetividade do tratamento. Uma terapêutica pouco efetiva é quando os medicamentos não apresentam os efeitos que se esperam deles 36 . Trata-se de uma questão mais difícil de ser investigada porque existem múltiplas variáveis que podem influenciar a inefetividade - indicação incorreta, posologia inadequada, via de administração errada, interações medicamentosas, modo de armazenamento, entre outros 37,38 .

Apenas em cerca de $57 \%$ e de $21 \%$ dos casos, no banco de artigos e equipamentos e de medicamentos, respectivamente, foram adotadas providências para evitar novos incidente similares, como a suspensão do uso do artigo/equipamento ou do medicamento. Adotar novos processos de cuidado após o ocorrido é fundamental para que sejam prevenidas novas ocorrências 25 . A Aliança Mundial para a Segurança do Paciente descreve diferentes tipos de investigação para melhorar a segurança do paciente e prevenir os possíveis danos, no uso de um ciclo de investigação que abrange: determinar a magnitude do dano, o número e tipos de eventos adversos que prejudicam os pacientes; entender as causas fundamentais dos danos ocasionados aos pacientes; identificar soluções para alcançar uma atenção à saúde mais segura e avaliar o impacto das soluções em situações da vida real 39.

\section{Conclusão}

A pesquisa apresentou limitações, dentre elas, as inerentes ao uso dos sistemas de notificação voluntária, com expressivo percentual de incompletude dos dados. Contudo, é importante destacar que a análise das notificações voluntárias permite a identificação de falhas ativas e, especialmente, das falhas latentes. E com essa identificação, torna-se possível recomendar a adoção de medidas para prevenir outros incidentes ou reduzir a sua gravidade.

Também cumpre frisar que, enquanto outros países envidam esforços para montar sistemas semelhantes, o desafio que se coloca para o NOTIVISA é o seu aprimoramento que, como em todos os sistemas de informação, decorre do uso, da crítica e da relação com os usuários - notificadores e interessados, tais como as instituições de pesquisa e ensino. Especificamente para o NOTIVISA, necessário também é que se produza uma mudança cultural nos potenciais notificadores quanto a informar sobre incidentes sem dano e eventos adversos para promover a melhoria contínua da segurança e qualidade dos serviços.

O estudo desvela notificações de eventos adversos e de incidentes que não causaram dano, provocando discussões do que efetivamente é entendido como dano para aquele que está notificando. Tais definições ainda a serem apropriadas pelo conjunto dos profissionais interessados na melhoria do cuidado requerem o aprofundamento de discussões para não somente o aprimoramento dos estudos sobre essa temática, mas também para o entendimento de quem executa a notificação.

Entre os problemas com a qualidade no cuidado, destacam-se o manejo do cateter central de inserção periférica e os erros de dosagem de medicamentos, sobretudo em recém-nascido no período neonatal precoce, a primeira semana e a mais crítica na vida de um recém-nascido. Frente aos achados, é imprescindível rever o uso e o manejo desses cateteres nos serviços de saúde, assim como todo o processo do uso medicamentoso, sobretudo os cálculos de dosagem para recém-nascido.

Desvelar os incidentes notificados faz parte de uma reflexão sobre a prática assistencial nas unidades neonatais nacionais e fomenta discussões para a melhoria do cuidado fornecido aos recém-nascido. A melhoria desse cuidado - juntamente com a melhoria da atenção prestada à gestante e ao parto - pode contribuir para a necessária redução do componente neonatal da mortalidade infantil. 


\section{Colaboradores}

L. S. Lanzillotti trabalhou na confecção da metodologia, busca do banco de dados, confecção das tabelas, análise dos resultados, discussão, conclusão e revisão do artigo. C. L. T. Andrade trabalhou na confecção das tabelas, na análise dos resultados e revisão do artigo. W. Mendes trabalhou na análise dos resultados, discussão, conclusão e revisão do texto. M. H. De Seta trabalhou na concepção do artigo, metodologia, análise dos resultados, discussão, conclusão e revisão do texto.

\section{Agradecimentos}

Agradecemos à Agência Nacional de Vigilância Sanitária (Anvisa), sobretudo ao Dr. Leandro Roberto da Silva atuante do Núcleo de Gestão do Sistema Nacional de Notificação e Investigação em Vigilância Sanitária (NUVIG), que disponibilizou gentilmente os bancos de dados para análise.

\section{Referências}

1. Secretaria de Vigilância em Saúde, Ministério da Saúde. Mortalidade materna no Brasil: principais causas de mortes e tendências temporais no período de 1990 a 2010. Brasília: Ministério da Saúde; 2011.

2. Gomes MASM, Lopes JMA, Moreira MEL, Gianini NOM. Assistência e mortalidade neonatal no setor público do Município do Rio de Janeiro, Brasil: uma análise do período 1994/2000. Cad Saúde Pública 2005; 21:1269-77.

3. Silva CF, Leite AJM, Almeida NMAGS, Ponce de Leon ACM, Olin I; Rede Norte-Nordeste de Saúde Perinatal. Fatores associados ao óbito neonatal de recém-nascidos de alto risco: estudo multicêntrico em Unidades Neonatais de Alto Risco no Nordeste brasileiro. Cad Saúde Pública 2014; 30:355-68.

4. Lanzillotti LS, Seta MH De, Andrade CLT, Mendes W. Eventos adversos e outros incidentes na unidade de terapia intensiva neonatal. Ciênc Saúde Coletiva 2015; 20:937-46.

5. Wolrd Health Organization. The conceptual framework for the international classification. Final Technical report January 2009. Geneva: World Health Organization; 2009
6. Wachter RM. Compreendendo a segurança do paciente. Porto Alegre: Editora Artmed; 2010.

7. Murff HJ, Patel VL, Hripcsak G, Bates DW. Detecting adverse events for patient safety research: a review of current methodologies. J Biomed Inform 2003; 36:131-43.

8. Larizgoitia I, Bouesseau M-C, Kelley E. WHO efforts to promote reporting of adverse events and global learning. J Public Health Res 2013; 2:e29.

9. Reis CT, Martins M, Laguardia J. A segurança do paciente como dimensão da qualidade do cuidado de saúde: um olhar sobre a literatura. Ciênc Saúde Coletiva 2013 ; 18:2029-36.

10. Ministério da Saúde. Portaria no 1.660, de 22 de Julho de 2009. Institui o Sistema de Notificação e Investigação em Vigilância Sanitária - VIGIPOS, no âmbito do Sistema Nacional de Vigilância Sanitária, como parte integrante do Sistema Único de Saúde - SUS. Diário Oficial da União 2009; 24 jul.

11. Agência Nacional de Vigilância Sanitária. Sistema de Notificação em Vigilância Sanitária - apresentação. http://www.anvisa.gov.br/hotsite/notivisa/ apresenta.htm (acessado em 10/Jan/2014). 
12. Torres ADE, Mota ELA. Notificação de eventos adversos em vigilância sanitária: Incompletude das variáveis do NOTIVISA em 2007 e 2008. Cad Saúde Coletiva 2010; 8:133-43.

13. Silva JPB, Santos DC, Meireles HAS, Souza FM, Aguiar ASS, Melgaço TB, et al. Análise do Sistema de Notificações em Vigilância Sanitária. Rev Para Med (Impr.) 2012; 26(4). http://files.bvs.br/upload/ S/0101-5907/2012/v26n4/a3478.pdf.

14. Oliveira JR, Xavier RMF, Santos Júnior AF. Eventos adversos notificados ao Sistema Nacional de Notificações para a Vigilância Sanitária (NOTIVISA): Brasil, estudo descritivo no período 2006 a 2011. Epidemiol. Serv Saúde 2012; 22:671-8.

15 Romero DE, Cunha CB. Avaliação da qualidade das variáveis socioeconômicas e demográficas dos óbitos de crianças menores de um ano registrados no Sistema de Informações sobre Mortalidade do Brasil (1996/2001). Cad Saúde Pública 2006; 22:673-84.

16. Agência Nacional de Vigilância Sanitária. Rede Sentinela: histórico. http://portal.anvisa.gov.br/ wps/content/anvisa+portal/anvisa/pos+-+comer cializacao+-+pos+uso/rede+sentinela/assunto+ $\mathrm{de}+$ interesse/noticias/rede+sentinela $+\mathrm{e}+$ desta que+em+lancamento+de+programa (acessado em 10/Fev/2014).

17. Agência Nacional de Vigilância Sanitária. Resolução RDC no 36, de 25 de julho de 2013. Institui ações para a segurança do paciente em serviços de saúde e dá outras providências. Diário Oficial da União 2013; 26 jul.

18. Harada MJCS, Pedreira MLG. Terapia intravenosa e infusões. São Caetano do Sul. São Paulo: Yendis Editora; 2011.

19. Rodrigues EC. Perdeu a veia: os significados da prática da terapia intravenosa em uma unidade de terapia intensiva neonatal do Rio de Janeiro. Rio de Janeiro [Tese de Doutorado]. Rio de Janeiro: Instituto Fernandes Figueira, Fundação Oswaldo Cruz; 2008.

20. Modes PSSA, Gaíva MAM, Rosa MKO, Granjeiro CF. Cuidados de enfermagem nas complicações da punção venosa periférica em recém-nascidos. Rev RENE 2011; 12:324-32.

21. Domingues G, Moraes FRRL, Júnior MAF. Tempo de permanência dos cateteres venosos periféricos e seus riscos para flebite relacionado ao sítio de inserção. Revista Científica Linkania Júnior 2012; 2:1-10.

22. Paulson PR, Miller KM. Neonatal peripherally inserted central catheters: recommendations for prevention of insertion and postinsertion complications. Neonatal Netw 2008; 27:245-57.

23. Vidal V, Jacquier A, Monnet O, Varoquaux A, Corroller TLE, Gaubert JY, et al. Cathéters centraux insérés par voie périphérique: une technique prometteuse. J Radiol 2008; 89:907-9.

24. Jesus VC, Secoli SR. Complicações acerca do cateter venoso central de inserção periférica (PICC). Ciênc Cuid Saúde 2007; 6:252-60.

25. Lawn JE, Enweronu-Laryea C, Massee Bateman O. Newborn survival in low resource settings- are we delivering? BJOG 2009; 116 Suppl 1:49-59.
26. Secretaria Municipal de Saúde do Rio de Janeiro. Taxa de mortalidade infantil, perinatal e fetal no Município do Rio de Janeiro - 1979 a 2002. Rio de Janeiro: Secretaria Municipal de Saúde do Rio de Janeiro; 2004.

27. Uauy R, Corvalan C, Casanello P, Kuzanovic J. Intervention strategies for preventing low birthweight in developing countries: importance of considering multiple interactive factors. Nestle Nutr Inst Workshop Ser 2013; 74:31-52.

28. Costa PQ, Lima JES, Coelho HLL. Prescrição e preparo de medicamentos sem formulação adequada para crianças: um estudo de base hospitalar. Braz J Pharm Sci 2009; 45:55-66.

29. Silva AEBC, Cassiani SHB. Administração de medicamentos: uma visão sistêmica para o desenvolvimento de medidas preventivas dos erros na medicação. Rev Eletrônica Enferm 2004; 6:279-85.

30. Tonello P, Andriguetti LH, Perassolo MS, Ziulkosk AL. Avaliação do uso de medicamentos em uma unidade pediátrica de um hospital privado do Sul do Brasil. Rev Ciênc Farm Básica Apl 2013; 34: 101-8.

31. Wegner W, Pedro ENR. Patient safety in care circumstances: prevention of adverse events in the hospitalization of children. Rev Latinoam Enferm 2012; 20:427-34.

32. Secretaria de Vigilância em Saúde, Ministério da Saúde. Programa Nacional de DST e AIDS. Manual de controle das doenças sexualmente transmissíveis. 4a Ed. Brasília: Ministério da Saúde; 2006.

33. Passos AF, Agostini FS. Conjuntivite neonatal com ênfase na sua prevenção. Rev Bras Oftalmol 2011; 70:57-67.

34. The United States Pharmacopeia. 21th Ed. Rockville: United States Pharmacopeial Convention; 1985.

35. Mandell GL, Bennett J, Dolin R. Principles and practice of infectious diseases: antimicrobial management cost and resistence. $6^{\text {th }}$ Ed. Philadelphia: Elsevier; 2005.

36. Godman A. Las bases farmacológicas de la terapéutica. Ciudad de México: Editorial McGraw-Hill Interamericana; 2003.

37. Agência Nacional de Vigilância Sanitária. Glossário e termos técnicos. http://www.anvisa.gov.br/farma covigilancia/conceito_glossario.htm (acessado em 14/Out/2012).

38. Capucho CH. Farmacovigilância hospitalar: processos investigativos em farmacovigilância. Pharmacia Brasileira. 2008. http://www.sbrafh.org.br/ site/public/temp/4f7baaa626c3a.pdf (acessado em 08/Jun/2015).

39. World Health Organization. La investigación en Seguridad del Paciente: mayor conocimiento para una atención más segura. Alianza Mundial para la Seguridad del Paciente. Geneva: World Health Organization; 2008. 


\section{Abstract}

This study aimed to analyze adverse events and near misses in newborns up to 28 days of life, reported to the Brazilian National Notification System for Sanitary Surveillance (NOTIVISA) system from 2007 to 2013. This was a quantitative, descriptive, retrospective study with analysis of secondary data. A total of 355 incidents were reported: 118 (33.3\%) related to medical devices, 4 (1.1\%) medical equipment, and 233 (65.6\%) medicines. Silver nitrate and antibiotics were the most frequently reported medicines, and among medical devices and equipment, phlebitis associated with IV lines was the most frequently reported adverse event. The study unveils the reporting of adverse events and near misses, fostering discussion on what actually constitutes harm according to the person that reports the event. The challenge for NOTIVISA is to improve the system, and as with other information systems, this results from its use, critical analysis, and interaction with users - incident reporters and interested parties like teaching and research institutions.

Patient Safety; Newborn Infant; Health Surveillance; Adverse Effects

\section{Resumen}

El estudio tuvo como objetivo analizar los eventos adversos y otros incidentes que no causaron daños en recién nacidos hasta sus 28 días de vida, notificados mediante Sistema de Notificación para Vigilancia de la Salud (NOTIVISA) durante los años de 2007 a 2013. Se trata de un estudio cuantitativo, descriptivo, retrospectivo, con un análisis de datos secundarios. Se notificaron 355 incidentes, de ellos, 118 (33,3\%) se relacionaron con artículos médicos, $4(1,1 \%)$ a equipamientos médicos, y 233 (65,6\%) a medicamentos. En lo que atañe a los medicamentos, el nitrato de plata y los antibióticos fueron los más notificados, y en los artículos y equipamientos médicos, el uso de catéteres venosos como precursores de flebitis, el evento adverso más notificado. El estudio desvela notificaciones de eventos adversos y de incidentes que no causaron daño, provocando discusiones de lo que efectivamente es considerado daño para quien realiza la notificación. El desafio para el NOTIVISA es su perfeccionamiento que, como en los demás sistemas de información, deriva del uso, crítica y relación con los usuarios - notificadores e interesados, tales como las instituciones de investigación yenseñanza.

Seguridad del Paciente; Recién Nacido;

Vigilancia Sanitaria; Efectos Adversos
Receb 25/Jun/2015

Versão final reapresentada em 09/Fev/2016

Aprovado em 23/Fev/2016 